\title{
Generation and Characterization of Cytochrome P450 2J3/10 CRISPR/Cas9 Knockout Rat Model
}

\author{
Jian Lu, Ang Chen, Xinrun Ma, Xuyang Shang, Yuanjin Zhang, Yuanqing Guo, Mingyao Liu, \\ and $\odot$ Xin Wang
}

Changning Maternity and Infant Health Hospital (J.L., Y.Z., Y.G., X.W.), and Shanghai Key Laboratory of Regulatory Biology, Institute of Biomedical Sciences and School of Life Sciences (J.L., A.C., X.M., X.S., Y.Z., M.L., X.W.), East China Normal University, Shanghai, People's Republic of China

Received May 10, 2020; accepted August 4, 2020

\begin{abstract}
Cytochrome P450 2J2 (CYP2J2) enzyme attracts more attention because it not only metabolizes clinical drugs but also mediates the biotransformation of important endogenous substances and the regulation of physiologic function. Although CYP2J2 is very important, few animal models are available to study its function in vivo. In particular, a CYP2J gene knockout (KO) rat model for drug metabolism and pharmacokinetics is not available. In this report, the CRISPR/Cas9 technology was used to delete rat CYP2J3/10, the orthologous genes of CYP2J2 in humans. The CYP2J3/10 KO rats were viable and fertile and showed no off-target effect. Compared with wild-type (WT) rats, the mRNA and protein expression of CYP2J3/10 in liver, small intestine, and heart of KO rats were completely absent. At the same time, CYP2J4 mRNA expression and protein expression were significantly decreased in these tissues. Further in vitro and in vivo metabolic studies of astemizole, a typical substrate of CYP2J, indicated that CYP2J was functionally
\end{abstract}

inactive in $\mathrm{KO}$ rats. The heart function indexes of $\mathrm{WT}$ and $\mathrm{KO}$ rats were also measured and compared. The myocardial enzymes, including creatine kinase-muscle brain type (CK-MB), creatine kinase (CK), and CK-MB/CK ratio, of KO rats increased by nearly $140 \%, 80 \%$, and $60 \%$, respectively. In conclusion, this study successfully developed a new CYP2J3/10 KO rat model, which is a useful tool to study the function of CYP2 $J$ in drug metabolism and cardiovascular disease.

\section{SIGNIFICANCE STATEMENT}

Human CYP2J2 is involved not only in clinical drug metabolism but also in the biotransformation of important endogenous substances. Therefore, it is very important to construct new animal models to study its function in vivo. This study successfully developed a new CYP2J knockout rat model by using CRISPR/Cas9 technology. This rat model provides a useful tool to study the role of CYP2J in drug metabolism and diseases.

\section{Introduction}

Human cytochrome P450 2J2 (CYP2J2) enzyme is involved in the metabolism of about $3 \%$ clinical drugs, which mainly include some antihistamines, such as astemizole, ebastine, and terfenadine (Xu et al., 2013; Zanger and Schwab, 2013). CYP2J2 is mainly expressed not only in human heart and liver (Askari et al., 2013; Lu et al., 2018) but also in tumors (Chen et al., 2011). In addition to drug metabolism, CYP2J2 is also involved in metabolism of endogenous substances such as arachidonic acid (AA), linoleic acid, eicosapentaenoic acid, docosahexaenoic acid, and vitamin D3 (Xu et al., 2013). AA is a polyunsaturated fatty acid with important physiologic functions (Chen et al., 2011; Xu et al., 2013). Epoxyeicosatrienoic acids (EETs) are derived from CYP2J2-mediated AA metabolism (Chen et al., 2011). CYP2J2 can

This work was supported in whole or part by grants from the National Natural Science Foundation of China [81301908, 81773808, and 81830083] and the Science and Technology Commission of Shanghai Municipality [17140901000, 17140901001, and 18430760400]. This work was also supported from East China Normal University Multifunctional Platform for Innovation [011].

https://doi.org/10.1124/dmd.120.000114. protect cardiac function in myocardial infarction-induced heart failure by increasing the concentration of circulating EETs and promoting angiogenesis through the Jagged1/Notch1 signaling pathway (Zhao et al., 2018). In addition, CYP2J2 inhibitor can significantly reduce the proliferation and migration and promote apoptosis of tumor cells by inhibiting the biosynthesis of EETs (Chen et al., 2011; Lu et al., 2018). Therefore, CYP2J2 plays a crucial role in drug metabolism and diseases.

In 2013, the Cyp $2 j$ locus was deleted through fusion bacterial artificial chromosome-mediated recombination, and the role of $C y p 2 j$ in hypoxic pulmonary vascular response was explored in this knockout mouse model (Zhou et al., 2013). In 2015, the role of CYP2J in pulmonary vascular remodeling and hypertension in mice was also discussed (Beloiartsev et al., 2015). The anti-inflammatory effect of CYP2J in macrophage is also revealed by a CYP2J4 knockout (KO) rat model (Behmoaras et al., 2015). However, a $C Y P 2 J 3 / 10 \mathrm{KO}$ animal model is not available. A $C Y P 2 J \mathrm{KO}$ rat model is important for pharmacological research, especially for drug metabolism and pharmacokinetic (DMPK) studies. Rats possess larger body size and have more body fluids, which are conducive to DMPK studies (Lu et al., 2017). Moreover, it is reported that the rat model shows advantages over the mouse model for the study of cardiovascular diseases (Iannaccone and Jacob, 2009; Zhao

ABBREVIATIONS: AA, arachidonic acid; Cas9, CRISPR-associated 9; CK, creatine kinase; CK-MB, creatine kinase-muscle brain type; $\mathrm{CL}_{\text {int }}$, intrinsic clearance; CYP2J2, cytochrome P450 2J2; DMPK, drug metabolism and pharmacokinetic; EET, epoxyeicosatrienoic acid; KO, knockout; LC-MS/MS, liquid chromatography-tandem mass spectrometry; P450, cytochrome P450; PCR, polymerase chain reaction; RLM, rat liver microsome; SD, Sprague-Dawley; sgRNA, single guide RNA; T7E1, T7 endonuclease 1; WT, wild-type. 
et al., 2018). As the third generation of artificial nuclease technology, the CRISPR/CRISPR-associated 9 (Cas9) system has become the most important gene editing tool (Shao et al., 2014; Wang et al., 2016). CRISPR/Cas9 has made an important breakthrough in the construction of transgenic animal models and targeted gene therapy (Shao et al., 2014). CRISPR/Cas9, as a new gene editing technology, has the characteristics and advantages of accurate targeting, simple operation, simultaneous knockout of multiple target genes, and no species restriction (Shao et al., 2014; Li et al., 2019). In recent years, scientists have made important progress in the construction of rat DMPK models by using the CRISPR/ Cas9 technology (Li et al., 2019). For example, in terms of rat DMPK models, we have successfully constructed Cyp2el (Wang et al., 2016), Cyp3a1/2 (Lu et al., 2017), Mdrla/b (Liang et al., 2019), and Slcolb2 (Ma et al., 2020) gene KO rats by using the CRISPR/Cas9 system. These rat DMPK models are further used in the study of drug metabolism and transport, pharmacodynamic, toxicity, and disease-related molecular mechanism, strengthening the relationship between DMPK and pharmacology/ toxicology, and promoting the new development of drug metabolism in innovative animal models (Qin et al., 2017; Ma et al., 2019).

In this report, the CRISPR/Cas9 technology is applied to the construction of the innovative rat $C Y P 2 J 3 / 10 \mathrm{KO}$ rat model, which can efficiently get the KO rats, significantly reduce the modeling cost, and shorten the modeling time. Further pharmacokinetic studies of astemizole, a typical substrate of CYP2J, confirmed that the KO rats lost the CYP2J metabolic function in vivo. This rat model provides a useful tool to study the role of CYP2J in drug metabolism and diseases.

\section{Materials and Methods}

Materials and Chemicals. Oligos (60 bp, containing CYP2J3 or CYP2J10 knockout target sites) and all primers for polymerase chain reaction (PCR) were ordered from Biosune Biotechnology Co. LTD. (Shanghai, China). Ethidium bromide was obtained from TianGen biotechnology Company (Beijing, China). Trizol, TA cloning kit, Prime Script RT Reagent Kit, SYBR Premix Ex Taq, and in vitro Transcription T7 Kit were bought from Takara (Dalian, China). The mMessage mMachine SP6 kit was bought from Thermo Scientific (Waltham, MA). ELISA kits for the detection of CYP2J3, CYP2J4, and CYP2J10 proteins were purchased from Jiangsu Meimian industrial Co., Ltd. (Jiangsu, China). Astemizole was bought from MeilunBio (Dalian, China), and $O$-desmethyl astemizole was purchased from Toronto Research Chemicals (Toronto, Canada). Glucose 6-phosphate, glucose 6-phosphate dehydrogenase, NADP, and tribromoethanol were bought from Sigma (St. Louis, MO). Tris base was purchased from Amresco (Solon, OH). The internal standard (mebendazole) for liquid chromatography-tandem mass spectrometry (LC-MS/MS) was purchased from Aladdin (Shanghai, China).

Animals. All wild-type (WT) male and female Sprague-Dawley (SD) rats used for gene targeting were purchased from the National Rodent Laboratory Animal Resources. All WT rats used in this research were littermate control of KO rats. Both WT and KO rats were kept under a specific pathogen-free facility with access to sterile water and rodent chow cubes, with 12-hour light-dark cycles. The research was conducted in accordance with the Declaration of Helsinki and/or with the Guide for the Care and Use of Laboratory Animals as adopted and promulgated by the US National Institutes of Health. All the animal experiment protocols in this study were approved by the Ethics Committee on Animal Experimentation of East China Normal University (Shanghai, China).

Generation of CYP2J3/10 Knockout Rat Model. The CYP2J3/10 KO rat model was generated through CRISPR/Cas9 system, and the gene targeting method was reported in our previous studies with some modifications (Wang et al., 2016; Lu et al., 2017). The sequence fragments of CYP2J3 and CYP2J10 were submitted to a CRISPR Design Tool (http://tools.genome-engineering.org) to get target sites [an $18 \mathrm{bp}$ DNA sequence with a protospacer adjacent motif site (5'-NGG-3') in the $3^{\prime}$ end]. Oligo DNA sequence (60 bp) containing 18 bp target sequence and $\mathrm{T} 7$ promoter was synthesized in vitro. By overlapping PCR, oligos (60 bp) were linked to the pGS3-T7-gRNA vector for single guide RNA (sgRNA) transcription. sgRNAs were produced by using $\mathrm{T} 7 \mathrm{Transcription} \mathrm{Kit} \mathrm{and} \mathrm{purified}$ with phenol/chloroform extraction method. Expression vectors for Cas9 were digested with NotI and then extracted by phenol/chloroform extraction methods. Cas9 mRNA was transcribed by using the mMessage mMachine SP6 kit and recovered by lithium chloride precipitation. The final concentrations of $C Y P 2 J 3$ sgRNA, CYP2J10 sgRNA, and Cas 9 mRNA for microinjection were $30 \mathrm{ng} / \mu \mathrm{l}$, $30 \mathrm{ng} / \mu \mathrm{l}$, and $50 \mathrm{ng} / \mu \mathrm{l}$, respectively. Cas 9 online designer (http://cas9.wicp.net/) was used to evaluate potential off-target sites for the two sgRNAs, and off-target sites with a score $\geq 0.25$ were selected for validation, using $\mathrm{T} 7$ endonuclease 1 (T7E1) assay. All primer pairs used in the generation of CYP2J KO rat model are listed in Table 1, including primer pairs for genotyping and off-target evaluation.

Real-Time PCR. Male SD rats (about 8 weeks) were sacrificed with carbon dioxide asphyxiation. Tissues were isolated as soon as possible and stored at $-80^{\circ} \mathrm{C}$ for further processing. All tissues were adequately ground under the atmosphere of liquid nitrogen and then transferred into tubes. Trizol was added into tubes for the lysis of tissues, and total mRNA was extracted according to the manufacturer's instructions. Total RNA was then reverse-transcribed into cDNA using the Takara RR036A RT kit. Real-time PCR was performed by using a Stratagene Mx3005P with SYBR Premix Ex Taq. All primer pairs used for real-time PCR are listed in Table 1. To detect the mRNA expression of CYP2J3, the primer pair (CYP2J3-QPCR forward and reverse) was designed on the downstream sequence of the sgRNA target site, and exon 1 to exon 3 of $C Y P 2 J 3$ gene were amplified. To detect the mRNA expression of CYP2J10, the primer pair (CYP2J10-Q-PCR forward and reverse) was designed on the downstream sequence of the sgRNA target site, and exon 1 to exon 2 of $C Y P 2 J 10$ gene were amplified. The PCR products for $C Y P 2 J 3$ and CYP2J10 mRNA detection were $268 \mathrm{bp}$ and $235 \mathrm{bp}$, respectively.

Enzyme-Linked Immunosorbent Assay. ELISA was conducted to detect the protein expression of CYP2J isoforms in rats. Male SD rats (about 8 weeks) were sacrificed with carbon dioxide asphyxiation. Tissues (liver, small intestine, and heart) were isolated as soon as possible and stored at $-80^{\circ} \mathrm{C}$ for further processing. PBS $(1 \mathrm{ml})$ was added into tissues $(100 \mathrm{mg})$, and then tissues were homogenized under the temperature of $4^{\circ} \mathrm{C}$. The homogenate was then centrifuged at $4^{\circ} \mathrm{C}, 3000 \mathrm{~g}$ for 20 minutes. The supernatant was collected for further use. The method of ELISA detection is according to the product instructions with some modification. Standard samples and test samples (tissues supernatant) were added into 96-well plate, respectively, and the enzyme-labeled reagent $(100 \mu \mathrm{l})$ was added into each well. Then, the plate was incubated at $37^{\circ} \mathrm{C}$ for 60 minutes and then washed with washing buffer for 5 times. Developer A $(50 \mu \mathrm{l})$ and developer B $(50 \mu \mathrm{l})$ were added into each well, and then the plate was shaken gently to mix, followed by incubation at $37^{\circ} \mathrm{C}$ for 15 minutes in the dark. After incubation, the reaction was stopped by adding the stop solution $(50 \mu \mathrm{l})$. Finally, the absorbance (optical density value) of each well was measured at $450 \mathrm{~nm}$ wavelength after setting the zero point by using the blank control well.

Metabolic Capacity of CYP2J in WT and KO Rats In Vitro. Methods for the preparation of rat liver microsomes (RLMs; from 8 weeks, male rats) were modified from our previous studies (Wang et al., 2010; Wang and Yeung, 2011). Astemizole was used as the probe substrate, and the reaction of astemizole $O$-demethylation was monitored for the evaluation of CYP2J metabolic activity in vitro ( $\mathrm{Lu}$ et al., 2018). The incubation mixture contained an NADPH regenerating system ( $5 \mathrm{mM}$ glucose 6-phosphate, $0.4 \mathrm{U} / \mathrm{ml}$ glucose 6-phosphate dehydrogenase, and $1 \mathrm{mM}$ NADP), astemizole $(0.2-10 \mu \mathrm{M})$, and $0.1 \mathrm{mg} / \mathrm{ml}$ of RLM in $0.05 \mathrm{M}$ Tris- $\mathrm{HCl}$ buffer (pH 7.4). The incubation was stopped by precooled acetonitrile after 20-minute incubation. The internal standard (mebendazole) was added, and the supernatant of the incubation mixture was then transferred to the autosampler vial after a protein precipitation process for LC-MS/ MS analysis. According to the standard Michaelis-Menten equation, $\mathrm{K}_{\mathrm{m}}$ and $\mathrm{V}_{\max }$ were analyzed by GraphPad Prism 6.0 (GraphPad Software Inc., San Diego, CA), and the $\mathrm{V}_{\max } / \mathrm{K}_{\mathrm{m}}$ ratio was defined as intrinsic clearance $\left(\mathrm{CL}_{\mathrm{int}}\right)$.

Pharmacokinetic Analysis of Astemizole in WT and KO Rats. Astemizole $(20 \mathrm{mg} / \mathrm{kg}$ ) was administered through gavage for all WT and KO rats (8 weeks, male), and blood samples $(300 \mu \mathrm{l})$ were collected into heparinized centrifuge tubes at $5,15,30,45,60,120,180,240,300$, and 420 minutes through caudal vein with capillary tubes. Blood samples were centrifuged at $5500 \mathrm{~g}$ for 15 minutes at $4^{\circ} \mathrm{C}$, and the plasma was transferred into new tubes and frozen at $-80^{\circ} \mathrm{C}$ for further detection. Protein precipitation was applied for the pretreatment of plasma samples. Acetonitrile $(300 \mu \mathrm{l})$ and internal standard (mebendazole, $10 \mu \mathrm{l})$ were added to $100 \mu \mathrm{l}$ plasma in order, followed by the centrifugation at $4^{\circ} \mathrm{C}, 14,000 \mathrm{~g}$ for 15 minutes. The supernatant $(70 \mu \mathrm{l})$ was transferred into the autosampler vial for LC-MS/MS detection. 
TABLE 1

Primer pairs used in this study

\begin{tabular}{|c|c|}
\hline Primer name & Primer sequence $\left(5^{\prime} \rightarrow 3^{\prime}\right)$ \\
\hline CYP2J3-genotyping-F & AACTGGACGGGTATGTAAC \\
\hline CYP2J3-genotyping-R & ACGCTCTATTGTGGCTTT \\
\hline CYP2J10-genotyping-F & AAATAGAAGTCAACACCACCAAT \\
\hline CYP2J10-genotyping-R & ACACGCAGGCAGGAGAAC \\
\hline CYP2J3-off target-1-F & GACССТTTCTTTCCCAGAC \\
\hline CYP2J3-off target-1-R & ATGTGACCGCACTTACCC \\
\hline CYP2J3-off target-2-F & ACTATGGGAGGCAAAGAC \\
\hline CYP2J3-off target-2-R & TGTGACAGTTACAGGTGGTT \\
\hline CYP2J3-off target-3-F & GCTGGCTTAAATACACTTGC \\
\hline CYP2J3-off target-3-R & AATGACTTCCTCCACATCCT \\
\hline CYP2J3-off target-4-F & TTCTGTCCTCACCCAAAG \\
\hline CYP2J3-off target-4-R & ACCCTCCTGTAAGCCACT \\
\hline CYP2J3-off target-5-F & ACAGGCTAGATTCAGACG \\
\hline CYP2J3-off target-5-R & AGTATTTGCTTTGGTGGG \\
\hline$C Y P 2 J 3$-off target-6-F & AAAGCCAGTATCCAGAAG \\
\hline CYP2J3-off target-6-R & GCTATGATGTTCCCAAGT \\
\hline CYP2J10-off target-1-F & TTCССАААТСТTТАССАТ \\
\hline CYP2J10-off target-1-R & AGGCTTGTTAATCATCCA \\
\hline CYP2J10-off target-2-F & TCACTCTGTTTGTGCCTTTA \\
\hline CYP2J10-off target-2-R & GCAGTATCCTGTCCACCTAT \\
\hline CYP2J3-Q-PCR-F & CCCTTAGTGGGTTGCTTG \\
\hline CYP2J3-Q-PCR-R & АССТССТTTGCTCСТтСC \\
\hline CYP2J4-Q-PCR-F & CTGGCCGATCGAGAATCCAT \\
\hline CYP2J4-Q-PCR-R & TGGTACCCTTTGGCAGGTGG \\
\hline CYP2J10-Q-PCR-F & CCGGCGTCCCAAGAACTA \\
\hline CYP2J10-Q-PCR-R & AGGTGTAACAGGGCGATT \\
\hline$\beta$-actin-Q-PCR-F & AGATCAAGATCATTGCTCCTCCT \\
\hline$\beta$-actin-Q-PCR-R & ACGCAGCTCAGTAACAGTCC \\
\hline
\end{tabular}

-F, forward; -R, reverse.

Methods for LC-MS/MS Analysis. For the LC-MS/MS detection, a 1290 HPLC6460 triple quadrapole mass spectrometer coupled with electrospray ionization ion source (Agilent Technologies) was employed. For the detection of astemizole and its metabolite $O$-demethylastemizole in both RLM incubation mixture and plasma, the mobile phase, consisting of water (A) with $0.1 \%$ formic acid and acetonitrile (B) with $0.1 \%$ formic acid, was applied. Astemizole and $O$-demethylastemizole were eluted by gradient with a flow rate of $0.3 \mathrm{ml} / \mathrm{min}$ as follows: $0-1$ minute, $20 \%$ acetonitrile; $1-3.5$ minutes, $20 \%-80 \%$ acetonitrile; 3.5-4.5 minutes, $80 \%$ acetonitrile; $4.5-5$ minutes, $80 \%-20 \%$ acetonitrile; 58 minutes, $20 \%$ acetonitrile. The detection of the ions was performed in a positive multiple reaction monitoring mode, monitoring the transition of $\mathrm{m} / \mathrm{z} 459.2-218.2$ for astemizole, $\mathrm{m} / \mathrm{z} 445.2-121.2$ for $O$-demethylastemizole, and m/z 296.1-264.1 for internal standard mebendazole.
Clinical Chemistry Analysis of WT and KO Rats. Blood from 8-week-old male rats was collected through caudal vein, and the serum was prepared by the following steps: standing at $4^{\circ} \mathrm{C}$ for 30 minutes, centrifugation at $4{ }^{\circ} \mathrm{C}, 1500 \mathrm{~g}$ for 15 minutes. Then the serum supernatant was transferred into a new tube and stored at $-80^{\circ} \mathrm{C}$ for further use. Serum samples were sent to ADICON Clinical Laboratories (Shanghai, China) for the detection of biochemical criterions, including albumin, globulin, albumin/globulin ratio, total proteins, alkaline phosphatase, aspartate aminotransferase, alanine aminotransferase, aspartate aminotransferase/alanine aminotransferase ratio, direct bilirubin, indirect bilirubin, total bilirubin, triglycerides, low-density-lipoprotein cholesterol, highdensity-lipoprotein cholesterol, total cholesterol, high-density-lipoprotein cholesterol/low-density-lipoprotein cholesterol ratio, creatine kinase (CK), creatine kinase-muscle brain type (CK-MB), and CK-MB/CK ratio.

Statistical Analysis. All data are presented as means \pm S.D. Statistical analysis between different groups was performed using two-tailed $t$ test, and $P$ values less than 0.05 were considered to indicate statistical significance. The pharmacokinetic parameters of astemizole were calculated by WinNonlin professional version 5.2.1 (Pharsight Mountain View, CA) based on the noncompartmental method.

\section{Results}

Generation of CYP2J3/10 Knockout Rat Model. According to the results from "CRISPR DESIGN", 5'-CCCCAAGAACTACCCGCC-3' (followed with AGG) and 5'-GGTGTAACAGGGCGATTC-3' (followed with AGG) were selected as target sites for rat $C Y P 2 J 3$ and CYP2J10, respectively. The quality of CYP2J3 and CYP2J10 sgRNA was good enough for injection, which was determined through agarose gel electrophoresis (Fig. 1, A and B). After coinjection of CYP2J3 sgRNA, CYP2J10 sgRNA, and Cas9 mRNA into one-cell fertilized eggs of SD rats, five progenies were born. For identification of gene modifications in the $\mathrm{F}_{0}$ generation progeny, sequence flanking target sites were amplified through PCR, and the products were digested with T7E1. For CYP2J3, PCR products of three progenies $\left(\mathrm{F}_{0}-2 \#, 3 \#, 4 \#\right)$ were detected with T7E1 cleavage, which indicated the potential of gene modification around the target sites (Fig. 1C). The PCR product was inserted into the $\mathrm{T}$ vector, and the sequencing of the $\mathrm{T}$ vector further revealed that all three progenies contained the same $10 \mathrm{bp}$ deletion at the target site (Fig. 1D). For CYP2J1O, amplification of sequence flanking the target site was not successful for $\mathrm{F}_{0}-3 \#, 5 \#$ until the product size was enlarged to more than $3 \mathrm{~kb}$. This demonstrated a large-scale deletion possibility, and direct sequencing of these products showed an $1199 \mathrm{bp}$

A
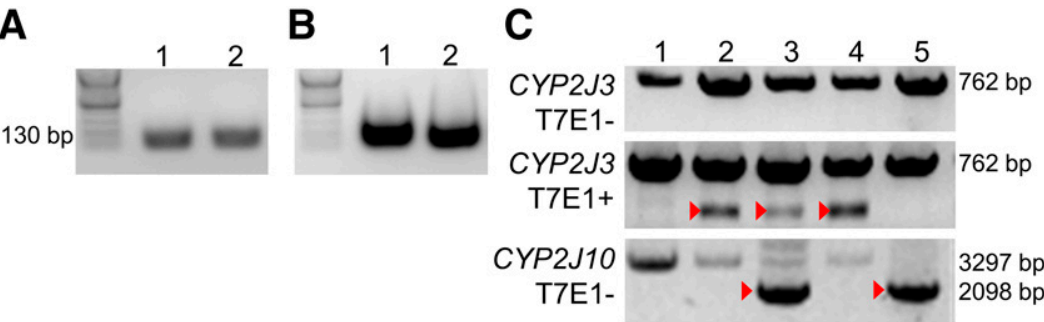

D

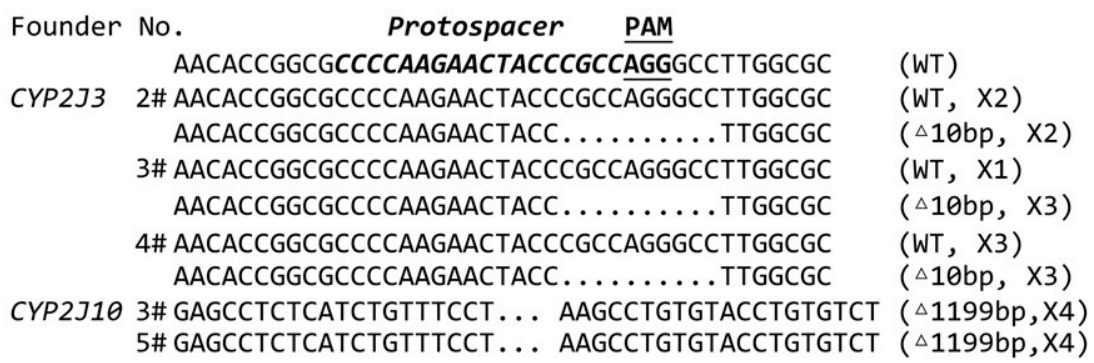

Fig. 1. Generation of $C Y P 2 J 3 / 10$ knockout rat model. (A) Characterization of precursors for $C Y P 2 J 3$ (lane 1) and CYP2J10 (lane 2) sgRNA. (B) Characterization of sgRNA for CYP2J3 (lane 1) and CYP2J10 (lane 2). (C) Detection of mutations in the $F_{0}$ generation using T7E1 assay. T7E1-, before T7E1 digestion; T7E1+, after T7E1 digestion; mutant band. (D) DNA sequencing flanking CYP2J3 and CYP2J10 target loci in $\mathrm{F}_{0}$ rats. ".", nucleotide deletion; " $\triangle$ ", the number of changed nucleotide; " $X$ ", the number of each genotype in four clones. 
TABLE 2

Potential off-target sites evaluated through T7E1

\begin{tabular}{|c|c|c|c|c|}
\hline Match name & Coordinate & Spacer + PAM & $\begin{array}{l}\text { No. of } \\
\text { mismatch }\end{array}$ & $\begin{array}{c}\text { Off-target } \\
\text { score }\end{array}$ \\
\hline CYP2J3 sgRNA & & CCCCAAGAACTACCCGCCAGG & & \\
\hline CYP2J3-OT-1 & chr11:39820711-39820729 & CCAAGgAaTACCCGCCA $\overline{G G}$ & 2 & 0.35 \\
\hline CYP2J3-OT-4 & chr6:125220805-125220824 & CCCAAGAAaaACCCGCC $\overline{\mathrm{AGG}}$ & 2 & 0.25 \\
\hline CYP2J3-OT-5 & chr4:144112581-144112600 & CCCAAGAAgaACCCGCC $\overline{A G G}$ & 2 & 0.25 \\
\hline СYР2J3-OT-6 & chr2:7454206-7454225 & CCCAAGAAgaACCCGCC $\overline{A G G}$ & 2 & 0.25 \\
\hline CYP2J10-OT-2 & chr2:116326415-116326400 & AACAGGGCGATTCA $\overline{G G}$ & 0 & 0.28 \\
\hline
\end{tabular}

OT, off-target site; PAM, protospacer adjacent motif.

deletion around the target site for both $\mathrm{F}_{0^{-}} 3 \#$ and $\mathrm{F}_{0^{-}}$-5\# (Fig. 1, C and D). Thus, $\mathrm{F}_{0}-3 \#$ was selected for the breeding of homozygous $\mathrm{KO}$ rats, and finally $\mathrm{F}_{2}$ progenies with $10 \mathrm{bp}$ deletion for $C Y P 2 J 3$ and $1199 \mathrm{bp}$ deletion for $C Y P 2 J 10$ were gained.

Off-Target Analysis. Off-target effects of CRISPR/Cas9 system, targeting CYP2J3 and CYP2J10, were searched on a genomewide sgRNA off-target searching tool (http://cas9.wicp.net/) and then evaluated by T7E1 cleavage assay. Off-target sites with a relatively high offtarget score $(\geq 0.25)$ were selected for T7E1 treatment (Table 2). Agarose gel electrophoresis results showed no off-target effect in all high potential off-target sites (Fig. 2).

Expression of CYP2J in Tissues of WT and KO Rats. The mRNA expression of $C Y P 2 J 3, C Y P 2 J 4$, and $C Y P 2 J 10$ in rat liver, small intestine, and heart was detected through specific primer pairs (Table 1). Agarose gel electrophoresis results verified that no mRNA expression of $C Y P 2 J 3$, $C Y P 2 \mathrm{~J} 4$, and $C Y P 2 \mathrm{~J} 10$ was detected in above tissues of $\mathrm{KO}$ rats, whereas it was highly expressed in WT control of these corresponding tissues (Fig. 3A).

The protein levels of $C Y P 2 J 3, C Y P 2 J 4$, and $C Y P 2 J 10$ in rat liver, small intestine, and heart were detected by ELISA. CYP2J3 was not detected in the liver of $\mathrm{KO}$ rats, whereas CYP2J4 and CYP2J4 decreased by about $80 \%$ and $90 \%$ (Fig. 3B), respectively. CYP2J3 and CYP2J10 in the small intestine of $\mathrm{KO}$ rats were undetectable, whereas CYP2J4 decreased by around $75 \%$ (Fig. 3B). In the heart of KO rats, CYP2J3, CYP2J4, and CYP2J10 decreased about 90\%, 60\%, and 90\% (Fig. 3B), respectively.

Metabolic Capacity of CYP2J in WT and KO Rats In Vitro. To assess the metabolic function of CYP2J in the $\mathrm{KO}$ rat in vitro, astemizole was chosen as a probe substrate to characterize the metabolic activity of CYP2J in RLMs. In the RLMs of WT rats, the $O$-desmethylastemizole was formed effectively, compared with that in RLMs of KO rats (Fig. 4, A-C). The apparent $K_{m}$ values for the metabolism of astemizole in the RLMs of WT and KO rats were 8.2 and $9.7 \mu \mathrm{M}$, respectively. The $\mathrm{V}_{\max }$ of astemizole metabolism in KO rats was decreased to $40 \%$ of that in WT rats, and the $\mathrm{CL}_{\text {int }}$ was also decreased to $35 \%$ of that in WT rats (Fig. 4, B and $\mathrm{C}$ ). All these results indicated that the CYP2J activity of KO rats was significantly decreased in vitro.

Metabolic Capacity of CYP2J in WT and KO Rats In Vivo. To further assess the metabolic function of CYP2J in the $\mathrm{KO}$ rat in vivo, the pharmacokinetics behavior of astemizole was monitored and compared between WT and KO rats. After oral administration of astemizole, the metabolic rate of $\mathrm{KO}$ rats was much slower, and the area under the curve (AUC) was 4.6 times higher than that of WT rats (Fig. 4, D and E, and Table 3). At the same time, the $\mathrm{C}_{\max }$ of astemizole in $\mathrm{KO}$ rats was significantly increased, with 2.4 times of that in WT rats (Table 3 ). CYP2J3/10 KO also prolonged the half-life (3.4 times of that in WT rats) and mean retention time (1.4 times of that in WT rats) and decreased the clearance $(\mathrm{CL} / \mathrm{F})$ and apparent volume of distribution $\left(\mathrm{V}_{\mathrm{d}} / \mathrm{F}\right)$ values (Table 3). Therefore, the pharmacokinetic results were consistent with the data of the in vitro metabolic activity test, indicating that CYP2J lost its metabolic function in $\mathrm{KO}$ rats.

Clinical Biochemical Criterion Analysis of WT and KO Rats. In addition to drug metabolism, CYP2J2 is also a great contributor to the biotransformation of physiologic substances and plays an important role in human health. The loss of $C Y P 2 J$ genes in rats may cause some physiologic changes, and thus the clinical chemistry analysis was carried out. Compared with WT rats, CYP2J3/10 KO in rats showed no significant difference of serum protein, alkaline phosphatase, transaminase, bilirubin, triglyceride, and cholesterol (Fig. 5A). Since CYP2J2 is necessary for cardiac function, the biomarkers of myocardial function were also measured and compared. The results showed that the values of CK-MB, CK, and CK-MB/CK ratio of $\mathrm{KO}$ rats significantly increased by almost $140 \%, 80 \%$, and $60 \%$, respectively, indicating that $\mathrm{KO}$ rat cardiomyocytes had the potential of injury (Fig. 5B).

A
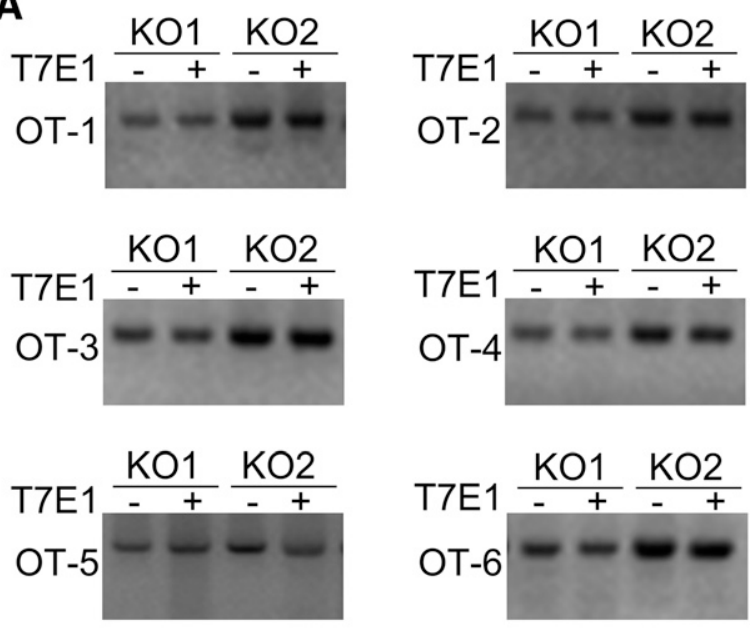

B
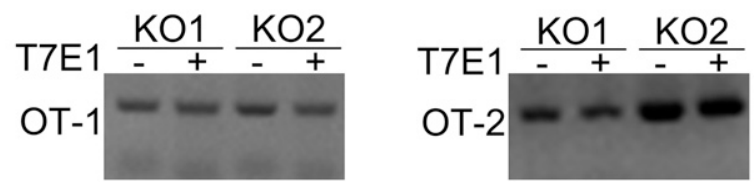

Fig. 2. Off-target analysis of $C Y P 2 J 3$ (A) and $C Y P 2 J 10$ (B) sgRNA. T7E1-, before T7E1 digestion; T7E1+, after T7E1 digestion; OT, off-target site. 
A

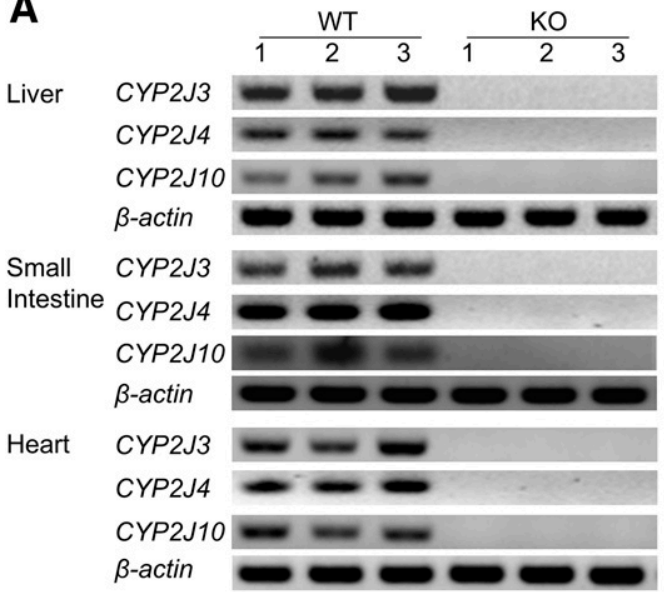

B

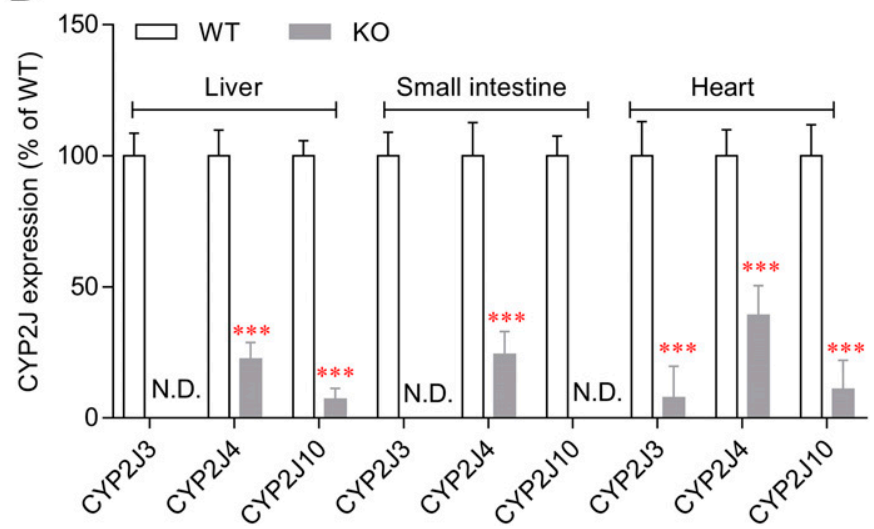

Fig. 3. The mRNA (A) and protein (B) expression of CYP2J3, CYP2J4, and CYP2J10 in liver, small intestine, and heart of WT and KO rats. N.D., not detected. The data are expressed as means \pm S.D. $(n=6) .{ }^{*} * *<0.001$ compared with WT group.

Compensatory Expression of Other Cytochrome P450 Isoforms and Nuclear Receptors in CYP2J KO Rats. The deficiency of CYP2J3/10 may affect the expression of other cytochrome P450 (P450) isoforms and nuclear receptors in rats. Thus, mRNA expression of other main $\mathrm{P} 450$ isoforms and nuclear receptors in the liver, small intestine, and heart were measured by real-time PCR. Cypla2 mRNA expression in the WT liver decreased by about $50 \%$ of that in the KO rat liver, whereas the expression of Cyp3a2 significantly increased by about $80 \%$ (Fig. 6A). No significant change was observed in the small intestinal expression of all tested $\mathrm{P} 450$ isoforms and nuclear receptors (Fig. 6B). In the heart tissue of KO rats, Cypla2, Cyp2e1, Cyp3a1, and Сур $3 a 2$ were upregulated by about $60 \%, 140 \%, 180 \%$, and $260 \%$, respectively (Fig. 6C).

\section{Discussion}

CYP2J2 is the only isoenzyme of the human CYP2J subfamily, whereas there are three CYP2J subtypes $(C Y P 2 J 3, C Y P 2 J 4$, and $C Y P 2 J 10$ ) in the rat genome. Compared with human $C Y P 2 J 2$ cDNA, the homology of $C Y P 2 J 3, C Y P 2 J 4$, and $C Y P 2 J 10$ cDNA is $79 \%, 80 \%$, and $78 \%$, respectively (data from National Center for Biotechnology Information). Human $C Y P 2 J 2$ is mainly expressed in the heart and liver, and the expression pattern of $C Y P 2 J 3$ in rats is similar (Xu et al., 2013). CYP2J10 is also expressed in rat aorta (Di Pascoli et al., 2015). However, $C Y P 2 J 4$ shows a different expression pattern compared with
A

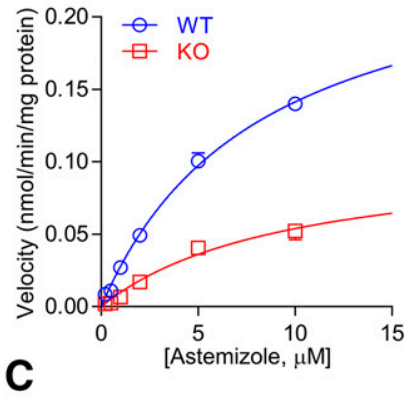

B

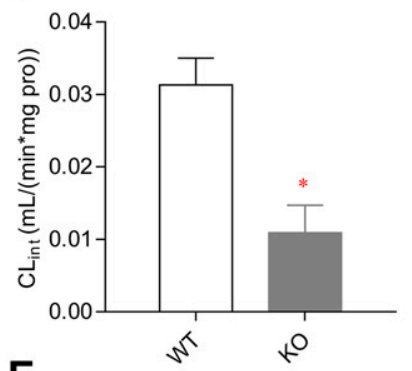

$\mathbf{E}$

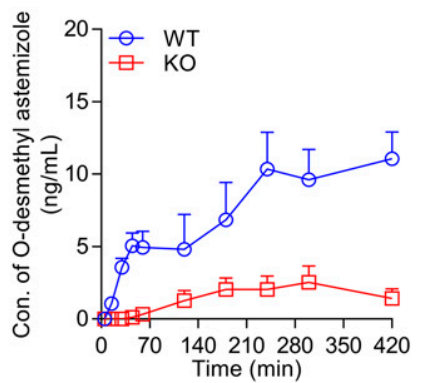

Fig. 4. Effects of CYP2J3/10 deletion on the metabolic capacity of CYP2J. (A) Saturation curve of astemizole metabolism in RLMs from WT and KO rats. (B) The $\mathrm{V}_{\max }$ of astemizole metabolism in RLMs from WT and KO rats. (C) The $\mathrm{CL}_{\text {int }}$ of astemizole metabolism in RLMs from WT and KO rats. (D) Pharmacokinetic profiles of astemizole in WT and KO rats. (E) The concentration-time curve of astemizole metabolite $(O$-desmethyl astemizole) in WT and KO rats. The data are expressed as means \pm S.D. $(n=5)$. $* P<0.05 ; * * * P<0.001$ compared with WT group.

human CYP2J2 (Zhang et al., 1998; Xu et al., 2013). Additionally, CYP2J3 shows similar metabolic preference to human $C Y P 2 J 2$ on some endogenous substance, such as AA (Xu et al., 2013). Therefore, $C Y P 2 J 3$ and $C Y P 2 J 10$ were selected in this study to investigate the effect of $C Y P 2 J$ deficiency on the metabolic functions in rats.

TABLE 3

Pharmacokinetic parameters of astemizole in WT and KO rats

All data are expressed as means \pm S.D. $(n=5)$

\begin{tabular}{lcc}
\hline Parameters & WT & KO \\
\hline $\mathrm{t}_{1 / 2}(\min )$ & $170.30 \pm 26.89$ & $582.40 \pm 155.30^{*}$ \\
$\mathrm{~T}_{\max }(\mathrm{min})$ & $57.00 \pm 30.89$ & $153.00 \pm 27.00^{*}$ \\
$\mathrm{C}_{\max }(\mathrm{ng} / \mathrm{ml})$ & $15.37 \pm 2.03$ & $36.25 \pm 7.60^{*}$ \\
$\mathrm{AUC}_{0-\mathrm{t}}(\mathrm{min} * \mu \mathrm{g} / \mathrm{ml})$ & $2.52 \pm 0.61$ & $11.50 \pm 2.56^{* *}$ \\
$\mathrm{~V}_{\mathrm{d}} / \mathrm{F}(\mathrm{L} / \mathrm{kg})$ & $1705.00 \pm 369.30$ & $626.90 \pm 124.10^{*}$ \\
$\mathrm{CL} / \mathrm{F}(1 / \mathrm{min}$ per kilogram) & $7.63 \pm 1.97$ & $0.88 \pm 0.23^{* *}$ \\
$\mathrm{MRT}(\mathrm{min})$ & $155.10 \pm 12.97$ & $210.70 \pm 2.76^{* *}$ \\
\hline
\end{tabular}

AUC, area under the curve; $C L$, clearance; MRT, mean retention time; $t_{1 / 2}$, half-life; $\mathrm{C}_{\max }$, maximum plasma concentration; $\mathrm{T}_{\max }$, time point at $\mathrm{C}_{\max } ; \mathrm{V}_{\mathrm{d}}$, apparent volume of distribution. $* * P<0.05 ; * * P<0.01$ compared with WT group. 


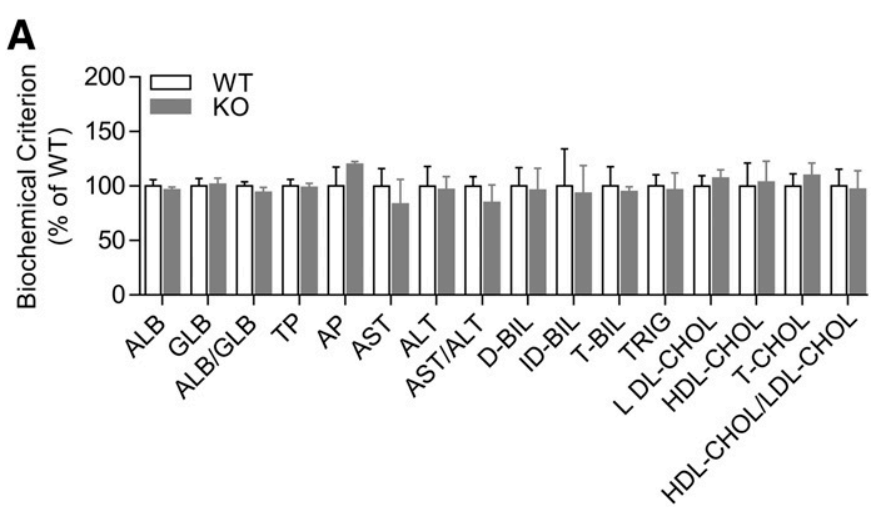

B
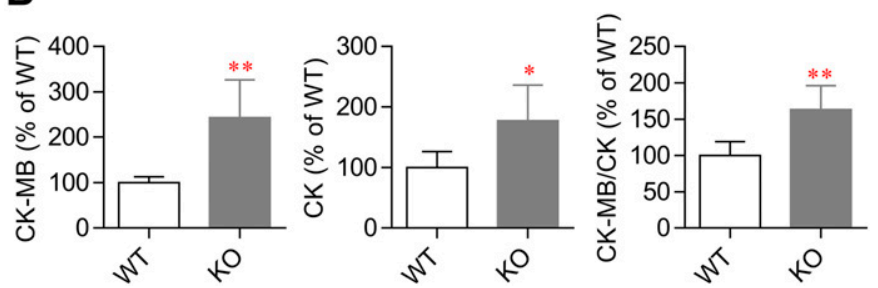

Fig. 5. Effects of $C Y P 2 J 3 / 10$ deletion on clinical biochemical criterions in $\mathrm{KO}$ rats. (A) Effects on serum protein, alkaline phosphatase, transaminase, bilirubin, triglyceride, and cholesterol. (B) Effects on creatine kinases. ALB, albumin; ALT, alanine amino transferase; AP, alkaline phosphatase; AST, aspartate amino transferase; D-BIL,direct bilirubin; GLB, globulin; HDL-CHOL, high-density-lipoprotein cholesterol; IDBIL, indirect bilirubin; LDL-CHOL, low-density-lipoprotein cholesterol; T-BIL total bilirubin; T-CHOL, total cholesterol; TP, total proteins; TRIG, triglycerides. All data are expressed as means \pm S.D. $(n=5)$. $* P<0.05 ; * * P<0.01$ compared with WT group.

To achieve effective target gene editing, we selected the target site at the first exon of $C Y P 2 J 3$ and finally obtained homozygotes with $10 \mathrm{bp}$ deletion around the target site, which causes frame shift mutation of $C Y P 2 J 3$. For $C Y P 2 J 10$ targeting, a preferential target was found and chosen at the second exon. However, the sgRNA targeting CYP2J10 tends to induce the large-scale deletion on both sides of the target site, resulting in homozygous CYP2J10 with 1199 bp deletion. The same phenomenon has been reported in previous studies; that is, even if only one sgRNA is introduced into the zygote, there may be a deletion extending to more than thousands of bases (Kosicki et al., 2018). It is reported that CRISPR/Cas9 system can tolerate a 1-3 base pair mismatch in its target sequence (Shao et al., 2014). Therefore, the off-target effects in $C Y P 2 J 3 / 10 \mathrm{KO}$ rat model were evaluated. Under the threshold value of 0.25 , only six and two potential off-target sites were selected for $C Y P 2 J 3$ and $C Y P 2 J 10$ sgRNA, respectively, which indicated a low off-target possibility of the target sites used in this study. No off-target effect was observed among these eight potential off-target sites by T7E1 assay. Furthermore, the PCR products were sequenced directly to verify the results from T7E1 assay (data not shown).

Our results showed the mRNA expression of $C Y P 2 J 3 / 10$ was completely absent in the liver, small intestine, and heart of $\mathrm{KO}$ rats. The protein level of CYP2J3 in the liver and small intestine of KO rats was undetectable, whereas it was detected in the heart of KO rats with a very low level ( $<10 \%$, compared with that in the WT group). Since the protein level of $C Y P 2 J 3$ was measured using ELISA, we speculate that the detected value in the heart of $\mathrm{KO}$ rats was the background value after the endogenous CYP2J3 was missing. Similarly, the protein level of CYP2J10 in the small intestine of KO rats was undetectable, and the levels in the liver and heart of $\mathrm{KO}$ rats were also very low $(<10 \%$, compared with that in the WT group). To evaluate the compensation of
$C Y P 2 J 4$ after $C Y P 2 J 3 / 10$ knockout, the mRNA and protein expression of $C Y P 2 \mathrm{~J} 4 \mathrm{in} \mathrm{KO}$ rats was measured. The mRNA expression of $C Y P 2 \mathrm{~J} 4$ was also completely absent in the liver, small intestine, and heart of $\mathrm{KO}$ rats. However, the protein expression of CYP2J4 in the liver, small intestine, and heart of $\mathrm{KO}$ rats decreased by $80 \%, 75 \%$, and $60 \%$, respectively. We speculate that $C Y P 2 \mathrm{~J} 4, C Y P 2 \mathrm{~J} 3$, and $C Y P 2 \mathrm{~J} 10$ share some regulatory regions and sequence because they are located in order on $5 \mathrm{q} 33$ of chromosome 5 and are close to each other. Therefore, the deletion of $C Y P 2 J 3$ and $C Y P 2 J 10$ sequences may interfere with the regulation region and sequence of $C Y P 2 J 4$ and eventually lead to the downregulation of $C Y P 2 J 4$. Our initial aim was to knock out $C Y P 2 J 3$ and $C Y P 2 J 10$, but we unexpectedly decreased the expression of $C Y P 2 J 4$. Such a model may be more conducive to study the function of CYP2J.

To further confirm the deficiency of metabolic function of CYP2J, its metabolic ability was evaluated both in vitro and in vivo. Although other P450 isoforms, such as CYP2D6 and CYP4F12, may be involved in the catalytic action of the conversion of astemizole to $O$-demethylated astemizole, CYP2J is still the primary drug metabolizing enzyme that mediates this reaction (Matsumoto and Yamazoe, 2001; Matsumoto et al., 2003; Xu et al., 2013). Therefore, astemizole is selected as the probe substrate of CYP2J metabolic capacity phenotype in rats (Matsumoto et al., 2003; Lu et al., 2018). The RLM incubation and pharmacokinetics of astemizole indicated that the metabolic function of

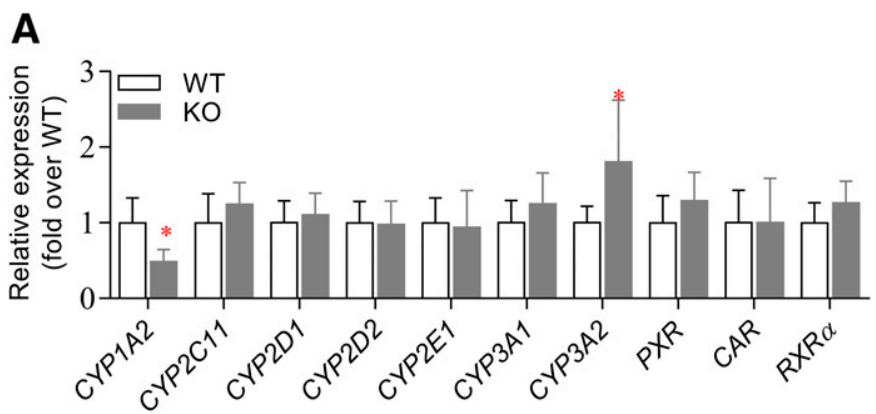

B

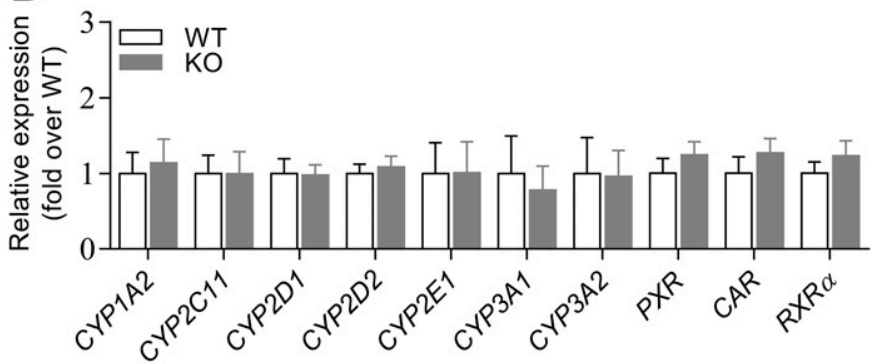

C

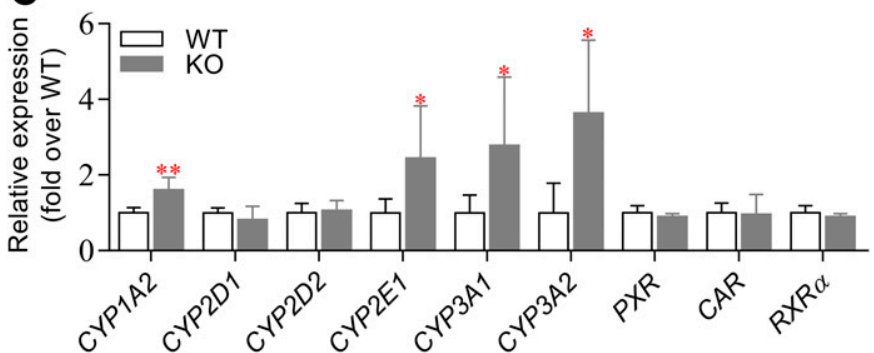

Fig. 6. Compensatory expression of other $\mathrm{P} 450$ isoforms and nuclear receptors in CYP2J3/10 KO rats. (A) Compensatory expression in the liver. (B) Compensatory expression in the small intestine. (C) Compensatory expression in the heart. All data are expressed as means \pm S.D. $(n=5) . * P<0.05 ; * * P<0.01$ compared with WT group. 
CYP2J was decreased in $\mathrm{KO}$ rats. The residual metabolic activity of $\mathrm{KO}$ group may be related to other $\mathrm{P} 450$ isoforms in rats. Therefore, the CYP2J3/10 KO rat model was generated successfully by using CRISPR/ Cas9 technology. To our knowledge, it is the first time to obtain CYP2J3/10 KO rats, which are a good tool to study CYP2J-mediated drug metabolism in vivo.

CYP2J is also involved in the metabolism of various endogenous substances and may play a role in physiologic functions. Thus, the basic physiologic status of KO rats was evaluated through clinical chemistry analysis. Compared with WT rats, CYP2J3/10 deficiency had no effect on serum protein, alkaline phosphatase, transaminase, bilirubin, triglyceride, and cholesterol. However, myocardial enzyme CK, CK-MB, and their ratio (CK-MB/CK) significantly increased by $140 \%, 80 \%$, and $60 \%$, respectively. Actually, the myocardial enzyme CK-MB is often used as a biomarker for the diagnosis of myocardial injury (Hueb et al., 2012) because it mainly exists in the heart muscle with relatively high concentration. In addition, the CK-MB index (CK-MB/CK) is a specific parameter to evaluate the relative contribution of cardiomyocyte damage, compared with other nonspecific myocardial cell injury (Saenger and Jaffe, 2007). In this study, CYP2J3/10 deficiency increased serum CK-MB concentration and the ratio of CK-MB/CK. These results suggested that there may be myocardial injury in $\mathrm{KO}$ rats, which needs further study.

AA and EETs, as essential polyunsaturated fatty acids, have many biologic functions, especially in human heart (Murray, 2016). CYP2J2 is a principal enzyme that promotes the biotransformation of AA to EETs in myocardial cells (Imig, 2012; He et al., 2017). In recent years, a series of studies have focused on the cardioprotection of the AA-CYP2J2-EET pathway (Ai et al., 2009; Imig, 2012; Ma et al., 2013; Fleming, 2014). In this study, the deletion of $C Y P 2 J 3 / 10$ was used to simulate the loss of function mutation of $C Y P 2 J 2$ in human body. The results revealed that $C Y P 2 J 3 / 10$ deficiency had some effects on cardiac function. Therefore, $C Y P 2 J 3 / 10$ is an important factor, not the only one, to maintain EET concentrations in vivo. Other P450s, such as CYP2C and CYP3A, have also been reported to mediate the biotransformation of AA to EETs (Panigrahy et al., 2010; Mitra et al., 2011). Therefore, the compensatory effect of other P450s in $\mathrm{KO}$ rats should be considered.

Last but not least, aging is a major risk factor for myocardial disease, which promotes changes in the structure and function of the heart, leading to deterioration in some patients with acute myocardial infarction (Boon et al., 2013; Roh et al., 2016). Therefore, we speculate that the effect of $C Y P 2 J 3 / 10$ deficiency on cardiac function of rats may become more and more serious in the process of aging. Further research should be carried out to make sure of this issue.

In conclusion, this study successfully developed a new $C Y P 2 J 3 / 10$ KO rat model, which provides a useful tool for the study of CYP2J in drug metabolism and diseases. CYP2J3/10 deficiency not only significantly reduced the metabolism of astemizole but also increased myocardial enzymes (CK and $\mathrm{CK}-\mathrm{MB}$ ) and their ratio (CK-MB/CK), which is a typical sign of myocardial injury. The present study provides a preliminary assessment of the role of CYP2J in drug metabolism and its potential impact on cardiac function.

\author{
Authorship Contributions \\ Participated in research design: Liu, Wang. \\ Conducted experiments: Lu, Chen, Ma, Shang, Zhang. \\ Performed data analysis: Lu, Wang. \\ Wrote or contributed to the drafting of the manuscript: Lu, Guo, Wang.
}

\section{References}

Ai D, Pang W, Li N, Xu M, Jones PD, Yang J, Zhang Y, Chiamvimonvat N, Shyy JY, Hammock $\mathrm{BD}$, et al. (2009) Soluble epoxide hydrolase plays an essential role in angiotensin II-induced cardiac hypertrophy. Proc Natl Acad Sci USA 106:564-569.

Askari A, Thomson SJ, Edin ML, Zeldin DC, and Bishop-Bailey D (2013) Roles of the epoxygenase CYP2J2 in the endothelium. Prostaglandins Other Lipid Mediat 107:56-63.

Behmoaras J, Diaz AG, Venda L, Ko JH, Srivastava P, Montoya A, Faull P, Webster Z, Moyon B, Pusey CD, et al. (2015) Macrophage epoxygenase determines a profibrotic transcriptome signature. J Immunol 194:4705-4716.

Beloiartsev A, da Glória Rodrigues-Machado M, Zhou GL, Tan TC, Zazzeron L, Tainsh RE, Leyton P, Jones RC, Scherrer-Crosbie M, and Zapol WM (2015) Pulmonary hypertension after prolonged hypoxic exposure in mice with a congenital deficiency of Cyp2j. Am J Respir Cell Mol Biol 52:563-570.

Boon RA, Iekushi K, Lechner S, Seeger T, Fischer A, Heydt S, Kaluza D, Tréguer K, Carmona G, Bonauer A, et al. (2013) MicroRNA-34a regulates cardiac ageing and function. Nature 495: $107-110$.

Chen C, Wei X, Rao X, Wu J, Yang S, Chen F, Ma D, Zhou J, Dackor RT, Zeldin DC, et al. (2011) Cytochrome P450 2J2 is highly expressed in hematologic malignant diseases and promotes tumor cell growth. J Pharmacol Exp Ther 336:344-355.

Di Pascoli M, Turato C, Zampieri F, Verardo A, Pontisso P, Pesce P, Sacerdoti D, and Bolognesi M (2015) Changes in gene expression of cytochrome P-450 in liver, kidney and aorta of cirrhotic rats. Prostaglandins Other Lipid Mediat 120:134-138.

Fleming I (2014) The pharmacology of the cytochrome P450 epoxygenase/soluble epoxide hydrolase axis in the vasculature and cardiovascular disease. Pharmacol Rev 66:1106-1140.

He Z, Yang Y, Wen Z, Chen C, Xu X, Zhu Y, Wang Y, and Wang DW (2017) CYP2J2 metabolites, epoxyeicosatrienoic acids, attenuate Ang II-induced cardiac fibrotic response by targeting $\mathrm{G \alpha}_{12 / 13}$. J Lipid Res 58:1338-1353.

Hueb W, Gersh BJ, Rezende PC, Garzillo CL, Lima EG, Vieira RD, Garcia RM, Favarato D, Segre CA, Pereira AC, et al.; MASS-V Study Group (2012) Hypotheses, rationale, design, and methods for prognostic evaluation of cardiac biomarker elevation after percutaneous and surgical revascularization in the absence of manifest myocardial infarction. A comparative analysis of biomarkers and cardiac magnetic resonance. The MASS-V Trial. BMC Cardiovasc Disord 12:65.

Iannaccone PM and Jacob HJ (2009) Rats! Dis Model Mech 2:206-210.

Imig JD (2012) Epoxides and soluble epoxide hydrolase in cardiovascular physiology. Physiol Rev 92:101-130.

Kosicki M, Tomberg K, and Bradley A (2018) Repair of double-strand breaks induced by CRISPR-Cas9 leads to large deletions and complex rearrangements. Nat Biotechnol 36: $765-771$

Li Y, Meng Q, Yang M, Liu D, Hou X, Tang L, Wang X, Lyu Y, Chen X, Liu K, et al. (2019) Current trends in drug metabolism and pharmacokinetics. Acta Pharm Sin B 9:1113-1144.

Liang C, Zhao J, Lu J, Zhang Y, Ma X, Shang X, Li Y, Ma X, Liu M, and Wang X (2019) Development and characterization of MDR1 (Mdrla/b) CRISPR/Cas9 knockout rat model. Drug Metab Dispos 47:71-79.

Lu J, Liu D, Zhou X, Chen A, Jiang Z, Ye X, Liu M, and Wang X (2018) Plant natural product plumbagin presents potent inhibitory effect on human cytochrome P450 $2 \mathrm{~J} 2$ enzyme. Phytomedicine 39:137-145.

Lu J, Shao Y, Qin X, Liu D, Chen A, Li D, Liu M, and Wang X (2017) CRISPR knockout rat cytochrome P450 3A1/2 model for advancing drug metabolism and pharmacokinetics research. Sci Rep 7:42922.

Ma B, Xiong X, Chen C, Li H, Xu X, Li X, Li R, Chen G, Dackor RT, Zeldin DC, et al. (2013) Cardiac-specific overexpression of CYP2J2 attenuates diabetic cardiomyopathy in male streptozotocin-induced diabetic mice. Endocrinology 154:2843-2856.

Ma X, Qin X, Shang X, Liu M, and Wang X (2019) Organic anion transport polypeptide 1b2 selectively affects the pharmacokinetic interaction between paclitaxel and sorafenib in rats. Biochem Pharmacol 169:113612.

Ma X, Shang X, Qin X, Lu J, Liu M, and Wang X (2020) Characterization of organic anion transporting polypeptide $1 \mathrm{~b} 2$ knockout rats generated by CRISPR/Cas9: a novel model for drug transport and hyperbilirubinemia disease. Acta Pharm Sin B 10:850-860.

Matsumoto S, Hirama T, Kim HJ, Nagata K, and Yamazoe Y (2003) In vitro inhibition of human small intestinal and liver microsomal astemizole O-demethylation: different contribution of CYP2J2 in the small intestine and liver. Xenobiotica 33:615-623.

Matsumoto S and Yamazoe Y (2001) Involvement of multiple human cytochromes P450 in the liver microsomal metabolism of astemizole and a comparison with terfenadine. $\mathrm{Br} \mathrm{J} \mathrm{Clin}$ Pharmacol 51:133-142.

Mitra R, Guo Z, Milani M, Mesaros C, Rodriguez M, Nguyen J, Luo X, Clarke D, Lamba J, Schuetz E, et al. (2011) CYP3A4 mediates growth of estrogen receptor-positive breast cancer cells in part by inducing nuclear translocation of phospho-Stat 3 through biosynthesis of $( \pm)-14,15$-epoxyeicosatrienoic acid (EET). J Biol Chem 286:17543-17559.

Murray M (2016) CYP2J2 - regulation, function and polymorphism. Drug Metab Rev 48:351-368 Panigrahy D, Kaipainen A, Greene ER, and Huang S (2010) Cytochrome P450-derived eicosanoids: the neglected pathway in cancer. Cancer Metastasis Rev 29:723-735.

Qin X, Lu J, Wang P, Xu P, Liu M, and Wang X (2017) Cytochrome P450 3A selectively affects the pharmacokinetic interaction between erlotinib and docetaxel in rats. Biochem Pharmacol 143:129-139.

Roh J, Rhee J, Chaudhari V, and Rosenzweig A (2016) The role of exercise in cardiac aging: from physiology to molecular mechanisms. Circ Res 118:279-295.

Saenger AK and Jaffe AS (2007) The use of biomarkers for the evaluation and treatment of patients with acute coronary syndromes. Med Clin North Am 91:657-681, xi.

Shao Y, Guan Y, Wang L, Qiu Z, Liu M, Chen Y, Wu L, Li Y, Ma X, Liu M, et al. (2014) CRISPR/Cas-mediated genome editing in the rat via direct injection of one-cell embryos. Nat Protoc 9:2493-2512.

Wang X, Cheung CM, Lee WY, Or PM, and Yeung JH (2010) Major tanshinones of Danshen (Salvia miltiorrhiza) exhibit different modes of inhibition on human CYP1A2, CYP2C9, CYP2E1 and CYP3A4 activities in vitro. Phytomedicine 17:868-875.

Wang X, Tang Y, Lu J, Shao Y, Qin X, Li Y, Wang L, Li D, and Liu M (2016) Characterization of novel cytochrome P450 2E1 knockout rat model generated by CRISPR/Cas9. Biochem Pharmacol 105:80-90.

Wang X and Yeung JH (2011) Effects of Salvia miltiorrhiza extract on the liver CYP3A activity in humans and rats. Phytother Res 25:1653-1659. 
Xu M, Ju W, Hao H, Wang G, and Li P (2013) Cytochrome P450 2J2: distribution, function, regulation, genetic polymorphisms and clinical significance. Drug Metab Rev 45:311-352.

Zanger UM and Schwab M (2013) Cytochrome P450 enzymes in drug metabolism: regulation of gene expression, enzyme activities, and impact of genetic variation. Pharmacol Ther 138:103-141. Zhang QY, Raner G, Ding X, Dunbar D, Coon MJ, and Kaminsky LS (1998) Characterization of the cytochrome P450 CYP2J4: expression in rat small intestine and role in retinoic acid biotransformation from retinal. Arch Biochem Biophys 353:257-264.

Zhao Q, Huang J, Wang D, Chen L, Sun D, and Zhao C (2018) Endothelium-specific CYP2J2 overexpression improves cardiac dysfunction by promoting angiogenesis via Jagged1/Notch1 signaling. J Mol Cell Cardiol 123:118-127.
Zhou GL, Beloiartsev A, Yu B, Baron DM, Zhou W, Niedra R, Lu N, Tainsh LT, Zapol WM, Seed $\mathrm{B}$, et al. (2013) Deletion of the murine cytochrome P450 Cyp2j locus by fused BAC-mediated recombination identifies a role for Cyp $2 \mathrm{j}$ in the pulmonary vascular response to hypoxia. PLoS Genet 9:e1003950.

Address correspondence to: Dr. Xin Wang, Changning Maternity and Infant Health Hospital, School of Life Sciences, East China Normal University, Shanghai 200241, China. E-mail: usxinwang@gmail.com; and xwang@bio.ecnu.edu.cn 